\title{
On the equation of motion of compact binaries in Post-Newtonian approximation
}

\author{
Yousuke Itoh \\ Max Planck Institut für Gravitationsphysik, Albert Einstein Institut \\ Am Mühlenberg 1, Golm 14476, Germany \\ E-mail: yousuke@aei.mpg.de
}

\begin{abstract}
A third post-Newtonian (3 PN) equation of motion for two spherical compact stars in a harmonic coordinate has been derived based on the surface integral approach and the strong field point particle limit. The strong field point particle limit enables us to incorporate a notion of a self-gravitating regular star into general relativity. The resulting $3 \mathrm{PN}$ equation of motion is Lorentz invariant, unambiguous, and conserves an energy of the binary orbital motion.
\end{abstract}

PACS numbers: 04.25.Nx,04.25.-g 


\section{Introduction}

Recently, high order post-Newtonian iteration for inspiralling compact binaries has attracted a renewed attention in the context of the worldwide gravitational waves detection efforts [1, 2, 3, 4,. Detectability and qualities of measurements of gravitational waves emitted by such binaries depend on our theoretical knowledge about waveforms and hence partly about dynamics of binaries [5].

The currently available equation of motion and the Hamiltonian that govern dynamics of (comparable masses) binaries in the post-Newtonian approximation of general relativity are the $3.5 \mathrm{PN}$ equations of motion in a harmonic coordinate [6, 7, 8] and the 3.5 PN Arnowitt-Deser-Misner (ADM) Hamiltonian in the ADM transversetraceless (ADMTT) gauge [9, 10, 11]. For reviews on post-Newtonian iteration, we refer readers to, e.g., [12.

In the inspiralling phase of spherical compact stars binaries, a point particle description is certainly useful and suitable to study their dynamics. A simple realization of this description may be a use of Dirac delta distributions, however, it causes ill-defined divergences due to the non-linearity of general relativity. The above mentioned works (except for [8]) have used Dirac delta distributions and resorted to regularization procedures such as Hadamard's partie finie. Then a problem has occurred. At the $3 \mathrm{PN}$ order, there exists one arbitrary undetermined parameter which reduces the predictability of the theoretical waveforms. Jaranowski and Schäfer [10, 13] who first achieved the $3 \mathrm{PN}$ iteration found two undetermined coefficients; $\omega_{\mathrm{k}}$ and $\omega_{\mathrm{s}}$ in their Hamiltonian (later they fixed $\omega_{\mathrm{k}}$ by imposing Poincaré invariance on their Hamiltonian [14]). Blanchet and Faye [6, 7] found one coefficient, called $\lambda$, in their equation of motion. They then showed [15, 16] that the results of these two independent studies are consistent with each other when the following relation holds; $\omega_{\mathrm{s}}=-11 \lambda / 3-1987 / 840$. It was suggested that unsatisfactory features of the regularizations they have used associated with their use of Dirac delta distributions were possible origins of the parameters, as summarized in [11.

In fact, Damour, Jaranowski, and Schäfer succeeded in deriving the complete 3 PN ADM Hamiltonian in the ADMTT gauge and fixed the parameter as $\omega_{\mathrm{s}}=$ 0 using dimensional regularization [11. However, applicability of mathematical regularizations to the current problem is not a trivial issue, but an assumption to be verified, or at least supported by convincing arguments. At the $2.5 \mathrm{PN}$ order, there is a physical argument of the "Dominant Schwarzschild Condition" 17] which supports a use of Dirac delta distributions and a certain sort of regularizations. There is no such argument at the $3 \mathrm{PN}$ order. Thus, it seems crucial to achieve unambiguous $3 \mathrm{PN}$ iteration without introducing singular sources and support the result of [11.

The author, Futamase and Asada have recently developed yet another method of derivation of a post-Newtonian equation of motion in a harmonic coordinate appropriate to inspiralling compact binaries [18, 19] which is based on the strong field point particle limit [20] and the surface integral approach [21]. In this paper, due to the lack of space, we briefly review our method and present few of our 3 PN results. Full explanations on our method and results will be reported elsewhere 22, 23].

\section{Strong field point particle limit}

We shall write the post-Newtonian expansion parameter explicitly as $\epsilon$, which represents smallness of the orbital velocity, $O(\epsilon)$, and weakness of the interstars field, 
$O\left(\epsilon^{2}\right)$. Futamase [20] advocated the strong field point particle limit where the radius of the star shrinks at the same rate as the mass in the post-Newtonian limit $(\epsilon \rightarrow 0)$. Thus, the typical scale of gravitational field inside the star $(\sim$ the mass over the radius of the star) remains finite (and can be strong) in $\epsilon$ zero limit. This is in contrast with the usual post-Newtonian expansion for an extended star, where the matter density is assumed to scale as $\epsilon^{2}$ and we apply the post-Newtonian approximation to the field even inside the stars.

\section{Field equations}

Using the post-Newtonian approximation, we iteratively solve the Einstein equations under the harmonic gauge condition that is written as $h^{\mu \nu}{ }_{, \nu}=0$ where $h^{\mu \nu} \equiv$ $\eta^{\mu \nu}-\sqrt{-g} g^{\mu \nu}, \eta^{\mu \nu} \equiv \operatorname{diag}\left(-\epsilon^{2}, 1,1,1\right)$ is the flat metric, and $g$ is the determinant of the metric $g_{\mu \nu}$. Then harmonically relaxed Einstein equations can be casted in integral forms as $h^{\mu \nu}\left(\tau, x^{i}\right)=4 \int_{C} d^{3} y \Lambda^{\mu \nu}\left(\tau-\epsilon|\vec{x}-\vec{y}|, y^{k} ; \epsilon\right) /|\vec{x}-\vec{y}|$, where $\Lambda^{\mu \nu} \equiv \Theta^{\mu \nu}+\chi^{\mu \nu \alpha \beta}{ }_{, \alpha \beta}, \Theta^{\mu \nu}$ is the matter plus the gravitational field stress energy pseudo-tensor $\left(\Theta^{\mu \nu} \equiv-g\left(T^{\mu \nu}+t_{L L}^{\mu \nu}\right)\right.$ with $t_{L L}^{\mu \nu}$ being the Landau-Lifshitz pseudo tensor) and $\chi^{\mu \nu \alpha \beta}{ }_{, \alpha \beta}$ arises since we use the wave operator of the flat spacetime instead of that of the curved spacetime. $\tau$ is the time coordinate. The light cone $C$ emanating from the field point $\left(\tau, x^{i}\right)$ is split into two zones; the near zone $N$ which encloses the two stars completely and the far zone $F$ which is the outside of $N$ and where the retardation effects of the field are manifest. To treat the retarded integrals over $F$ we have adopted the Direct Integration of the Relaxed Einstein equations method developed by Will and collaborators [8], however, it is well-known [8, 24] that the integrals over $F$ do not contribute to an equation of motion up to the $3.5 \mathrm{PN}$ order and hence we devote ourselves to the integrals over $N$.

The integral region $N$ then is split into three regions; two spheres, called the body zone $B_{A}(A=1,2$ labels each star), each of which surrounds each star with no intersection with the other nor the star, and elsewhere $N / B$ where $B \equiv B_{1} \cup B_{2}$. We make the radius of $B_{A}$ shrink proportionally to $\epsilon$ to define the multipole moments and to derive an equation of motion for compact stars. That is, $B_{A} \equiv\left\{x^{i}|| \vec{x}-\vec{z}_{A}(\tau) \mid \leq\right.$ $\left.\epsilon R_{A}\right\}$ where $\epsilon R_{A}$ is arbitrary but smaller than the orbital separation $r_{12}$ and larger than the radius of the star for any $\epsilon . z_{A}^{i}(\tau)$ represents the location of the star $A$ in the point particle limit. The integrals over each $B_{A}$ are evaluated using the multipole expansion of the star. For example, the ( $\Theta$ part of the) mass multipole moments of the star $A$ are defined as $I_{A \Theta}^{K_{l}} \equiv \epsilon^{2} \int_{B_{A}} d^{3} \alpha_{A} \Theta^{\tau \tau} \alpha \frac{K_{l}}{A}$, where $\alpha_{A}^{i} \equiv \epsilon^{-2}\left(x^{i}-z_{A}^{i}(\tau)\right)$ is a scaled coordinate in which the star does not shrink to the point $z_{A}^{i}(\tau)$. The capital index denotes a set of collective indices, $I_{l} \equiv i_{1} \cdots i_{l}$ and $\alpha \frac{I_{l}}{A} \equiv \alpha_{A}^{\underline{i}_{1}} \alpha_{A}^{\underline{i}_{2}} \cdots \alpha_{A}^{\underline{i}_{l}}$.

The remaining Poisson-type integrals over $N / B, \int_{N / B} d^{3} y|\vec{x}-\vec{y}|^{n-1} f(\tau, \vec{y})(n \geq 0$ : integer, $f(\tau, \vec{x})$ being a certain combination of $\left.\Lambda^{\mu \nu}(\tau, \vec{x})\right)$, are evaluated with the help of particular solutions of Poisson equations. For $n=0$ case, using $\Delta g(\vec{x})=f(\vec{x})$ (for brevity, we do not write $\tau$ dependence in $f$ nor $g$ here), we have

$$
\int_{N / B} \frac{d^{3} y f(\vec{y})}{|\vec{x}-\vec{y}|}=-4 \pi g(\vec{x})+\oint_{\partial(N / B)} d S_{k}\left[\frac{1}{|\vec{x}-\vec{y}|} \frac{\partial g(\vec{y})}{\partial y^{k}}-g(\vec{y}) \frac{\partial}{\partial y^{k}}\left(\frac{1}{|\vec{x}-\vec{y}|}\right)\right] .
$$

Once explicit expressions of the necessary particular solutions are in hand, it is straightforward to evaluate the Poisson-type integrals. 


\section{Mass-energy relation and momentum-velocity relation}

In our formalism, we do not assume a specific expression of the matter's stress energy tensor. Thus, we need to express the operationally defined multipole moments, namely, the four momentum $P_{A \Theta}^{\mu} \equiv \epsilon^{2} \int_{B_{A}} d^{3} \alpha_{A} \Theta^{\mu \tau}$ of the star in terms of the mass $m_{A}$, the velocity $\vec{v}_{A}$, and $\vec{r}_{12}$. As for $P_{A \Theta}^{\tau}$, we use its evolution equation which is derived by the local energy momentum conservation $\Theta^{\mu \nu}{ }_{, \nu}=0$ as $d P_{A \Theta}^{\tau} / d \tau=$ $-\epsilon^{-4} \oint_{\partial B_{A}} d S_{k}(-g) t_{L L}^{k \tau}+\epsilon^{-4} v_{A}^{k} \oint_{\partial B_{A}} d S_{k}(-g) t_{L L}^{\tau \tau}$. At the lowest order, we obtain $d P_{A \Theta}^{\tau} / d \tau=O\left(\epsilon^{2}\right)$. The evolution equation of $P_{A \Theta}^{\tau}$ is then solved functionally into a form $P_{A \Theta}^{\tau}=m_{A}\left[1+O\left(\epsilon^{2}\right)\right]$ where the mass of the star $A, m_{A}$, is defined as the integration constant by $m_{A} \equiv \lim _{\epsilon \rightarrow 0} P_{A \Theta}^{\tau}$. We note that although $m_{A}$ is defined by $\epsilon \rightarrow 0$ limit of $P_{A \Theta}^{\tau}$, the gravitational field energy inside the star is taken into account in the definition of $m_{A}$ since $P_{A \Theta}^{\tau}$ is defined as a volume integral whose integrand includes the field energy $(-g) t_{L L}^{\tau \tau}$ which does not vanish in the $\epsilon$ zero limit because of the scaling of the strong field point particle limit. (the mass over the radius of the star is finite inside the star independently of $\epsilon$ ). Following the Newtonian dynamics, the momentum-velocity relation on the other hand is derived by the time derivative of the dipole moment $D_{A \Theta}^{i} \equiv \epsilon^{2} \int_{B_{A}} d^{3} \alpha_{A} \Theta^{\tau \tau} \alpha_{A}^{\underline{i}}$ as $P_{A \Theta}^{i}=P_{A \Theta}^{\tau} v_{A}^{i}+Q_{A \Theta}^{i}+\epsilon^{2} d D_{A \Theta}^{i} / d \tau$ where $Q_{A \Theta}^{i} \equiv \epsilon^{-4} \oint_{B_{A}} d S_{k}\left((-g) t_{L L}^{\tau k}-v_{A}^{k}(-g) t_{L L}^{\tau \tau}\right) y_{A}^{i}=-\epsilon^{6} d\left(m_{A^{0}}^{3} a_{A}^{i} / 6\right) / d \tau$ ( ${ }_{0} a_{A}^{i}$ is the Newtonian acceleration) arises due to the non-compactness of $(-g) t_{L L}^{\mu \nu} \cdot Q_{A \Theta}^{i}$ appears starting from the $3 \mathrm{PN}$ order and it affects the $3 \mathrm{PN}$ equation of motion. Finally, we note that as in the Newtonian case, we can choose the value of the dipole moment $D_{A \Theta}^{i}$ freely to define the representative point of the star $A, z_{A}^{i}(\tau)$. For instance, $z_{A}^{i}(\tau)$ corresponding to $D_{A \Theta}^{i}=0$ may be called the center of mass of the star $A$. However, we found that it is convenient to adopt $D_{A \Theta}^{i}=\epsilon^{4}\left(1 / 6-22 \ln \left(r_{12} / \epsilon / R_{A}\right)\right) m_{A 0}^{3} a_{A}^{i}$ at the $3 \mathrm{PN}$ order 22,23 . Choosing a certain value for $D_{A \Theta}^{i}$ corresponds to choosing the corresponding coordinate under the harmonic gauge condition. Our particular choice gauges $\ln \epsilon R_{A}$ dependence away from the $3 \mathrm{PN}$ acceleration. A different choice leaves $\ln \epsilon R_{A}$ dependences in an acceleration, however, these are mere gauge terms and do not appear when we are concerned with coordinate invariant quantities. In fact, a different choice $D_{A \Theta}^{i}=\epsilon^{4} m_{A 0}^{3} a_{A}^{i} / 6$ gives the same invariant binary orbital energy of circular orbital motion in the center of mass frame as $E(x)$ given below [23].

\section{Equation of motion}

There may be three methods being studied in derivation of a high order postNewtonian equation of motion. The first one is the volume integral approach, which may take the following form in the Newtonian dynamics: $m_{A} d v_{A}^{i} / d \tau=$ $\int d^{3} y \rho_{A}(\vec{y}) \phi(\vec{y})^{, i}$. ( $\rho_{A}$ denotes the density of the star $A$. $\phi$ is the Newtonian potential.) In this method, one can track how the stars' internal structures dependent terms cancel out (or remain) and disappear (or appear) in the final result; if the resulting equation of motion depends on, say, the density profile of (almost stationary) spherically symmetric compact stars, it suggests violation of the equivalence principle to some extent. This method is being used by Pati and Will [8].

The second method assumes that a regularized action describes dynamics of binaries. Blanchet and Faye 6, 7] have used this method with the generalized Hadamard's partie finie regularization but obtained one arbitrary parameter $\lambda$ in their $3 \mathrm{PN}$ equation of motion. It is shown 6, 7] that a regularized geodesics can be 
derived from a certain action. This method is attractive, besides its mathematical beauties, because if the resulting equation coincides with the equations derived by the first (and the third below) method, then it suggests a notion of "geodesics in a dynamical spacetime" (with a certain class of regularizations supplemented).

The third method, which we have adopted, is the surface integral method where we compute the gravitational momentum flux $t^{i j}$ going through a sphere surrounding the star concerned. In the Newtonian case, we may have $m_{A} d v_{A}^{i} / d \tau=-\int d S_{j} t^{i j}$; $t^{i j}=1 /(4 \pi)\left(\phi^{, i} \phi^{, j}-\delta^{i j} \phi^{, k} \phi_{, k} / 2\right)$. In this method, possible information of the stars' internal structures are coded in, say, the multipole moments of the stars and hence it affects the equation of motion through $t^{i j}$. Based on the local energy momentum conservation, we obtain in our formalism a simple generalization of the surface integral approach to general relativity as

$$
\begin{aligned}
m_{A} \frac{d v_{A}^{i}}{d \tau} & =-\oint_{\partial B_{A}} d S_{k}(-g) t_{L L}^{k i}+v_{A}^{k} \oint_{\partial B_{A}} d S_{k}(-g) t_{L L}^{\tau i} \\
& +\left(m_{A}-P_{A \Theta}^{\tau}\right) \frac{d v_{A}^{i}}{d \tau}+\frac{d P_{A \Theta}^{\tau}}{d \tau} v_{A}^{i}-\frac{d Q_{A \Theta}^{i}}{d \tau}-\frac{d^{2} D_{A \Theta}^{i}}{d \tau^{2}}
\end{aligned}
$$

\section{Gravitational field and equations of motion up to the $3 \mathrm{PN}$ order}

Up to the $2.5 \mathrm{PN}$ order, it is possible to derive the gravitational field explicitly by the method described above. Once we have the field in closed form, it is straightforward to evaluate the surface integrals in Eq. (11). However, it does not seem possible to find explicitly all the particular solutions for the Poisson-type integrals over $N / B$ necessary to evaluate the $3 \mathrm{PN}$ gravitational field, even in the neighborhood of the star. We thus partly abandon derivation of the field and took an alternative method 22. We change the order of integration; for the integrands for which we could not find the particular solutions of Poisson equations, we first evaluate the surface integrals in Eq. (1) before evaluating the Poisson-type integrals and then evaluate the remaining volume integrals. With this method, we obtain a $3 \mathrm{PN}$ equation of motion. Because of the enormous length of the final result, we here present only the $3 \mathrm{PN}$ relative acceleration in the case of the circular orbit and in the center of mass frame, which is an appropriate equation to inspiralling binaries; $d V^{i} / d \tau=-\Omega^{2} r_{12}^{i}+\epsilon^{5}{ }_{2.5 \mathrm{PN}} A^{i}$ where $V^{i}=v_{1}^{i}-v_{2}^{i}$ is the relative velocity and ${ }_{2.5 \mathrm{PN}} A^{i}$ is the relative acceleration at the 2.5 $\mathrm{PN}$ order (the radiation reaction term). The $3 \mathrm{PN}$ orbital angular frequency $\Omega$ is,

$$
\begin{aligned}
m^{2} \Omega^{2} & =\gamma^{3}\left[1+\epsilon^{2} \gamma(-3+\nu)+\epsilon^{4} \gamma^{2}\left(6+\frac{41}{4} \nu+\nu^{2}\right)+\epsilon^{6} \gamma^{3}(-10\right. \\
& \left.\left.+\nu\left\{-\frac{2375}{24}+\frac{41 \pi^{2}}{64}\right\}+\frac{19}{2} \nu^{2}+\nu^{3}\right)\right]+O\left(\epsilon^{7}\right)
\end{aligned}
$$

where $m=m_{1}+m_{2}, \nu=m_{1} m_{2} / m^{2}, \gamma=m / r_{12}$. We here note that it is not allowed to fix the $\lambda$ parameter by comparing Eq. (2) with the corresponding result of Blanchet and Faye [6], since the harmonic gauge condition both groups have used does not fix a coordinate completely [23, 22].

Although we can not show it here due to the lack of space, our equation of motion respects the Lorentz invariance. And more importantly, our 3 PN equation of motion is unambiguous and admits a conserved energy. In the case of the circular orbit (neglecting the radiation reaction force) and in the center of mass frame, the invariant 
orbital energy $E$ becomes

$$
\begin{aligned}
& E(x)=-\frac{m \nu x}{2}\left[1+\epsilon^{2}\left(-\frac{3}{4}-\frac{1}{12} \nu\right) x+\epsilon^{4}\left(-\frac{27}{8}+\frac{19}{8} \nu-\frac{1}{24} \nu^{2}\right) x^{2}\right. \\
& \left.+\epsilon^{6}\left(-\frac{675}{64}+\left\{\frac{34445}{576}-\frac{205 \pi^{2}}{96}\right\} \nu-\frac{155}{96} \nu^{2}-\frac{35}{5184} \nu^{3}\right) x^{3}\right]+O\left(\epsilon^{7}\right),
\end{aligned}
$$

where $x=(m \Omega)^{2 / 3}$. We have thus derived a $3 \mathrm{PN}$ equation of motion free from an arbitrary parameter. Since we introduced $R_{A}$ in the definition of $B_{A}$, it may seem that $R_{A}$ appears in the field and also in the equation of motion. $B_{A}$ is introduced to separate the region where the presence of the star may make the field strong from well outside of the stars where the post-Newtonian approximation to the field is applicable. The contribution to the field from $B_{A}$ is evaluated with the multipole expansion. The equation of motion is derived via the surface integrals over $\partial B_{A}$. Now, as for $R_{A}$ dependent terms in the field, since we have used the non-singular matter sources, the field is smooth near $\partial B_{A}$ and possible $R_{A}$ dependences in the body zone contributions to the field cancel out the corresponding $R_{A}$ dependences in the $N / B$ contributions. As for an equation of motion, we have proved in [19] that an equation of motion derived from Eq. (1) does not depend on $R_{A}$. See [18, 19 for more details.

Comparing our $E(x)$ with that in [6], we determine the coefficient undetermined in the Blanchet and Faye 3 PN equation of motion as $\lambda=-1987 / 3080$. This value of $\lambda$ is consistent with the result of Damour, Jaranowski, and Schäfer 11. Thus, our result (indirectly) validates their use of dimensional regularization in the ADM Hamiltonian approach in the ADMTT gauge.

In the paper 22, 23, we devote ourselves to two spherical compact binaries, however, our formalism can be extended to include higher order multipole moments of the stars 18. We have used the strong field point particle limit and the surface integral approach to treat strongly self-gravitating stars. Therefore our 3 PN equation of motion is applicable to regular compact stars with strong self-gravity. As is clear from Eq. (2), our equation of motion depends only on $m_{A}$, and this fact supports the strong equivalence principle up to the $3 \mathrm{PN}$ order.

Finally, as mentioned above, we could not derive the complete $3 \mathrm{PN}$ gravitational field in closed forms. This fact makes it difficult to proceed to the $4 \mathrm{PN}$ iteration. The enormous numbers of terms in the intermediate computations, of order of $10^{5}$, appear even at the $3 \mathrm{PN}$ order. Brute force post-Newtonian iteration at the $4 \mathrm{PN}$ order may need efficient computations techniques. It may be possible to adopt the strong field point particle limit in the currently available waveform computation formalism and fix the undetermined parameters in the $3.5 \mathrm{PN}$ accurate waveforms.

\section{Acknowledgments}

The author would like to acknowledge T. Futamase and H. Asada for fruitful discussions and comments.

\section{References}

[1] Tagoshi H et al 2001 Phys. Rev. D63 062001

[2] Ando M et al 2001 Phys. Rev. Lett. 863950

[3] Takahashi H et al 2003 Preprint gr-qc/0307002 
[4] Abbott B et al 2003 Preprint gr-qc/0308069

[5] Cutler C et al. 1993 Phys. Rev. Lett. 702984

[6] Blanchet L and Faye G 2000 Phys. Lett. A271 58

[7] Blanchet L and Faye G 2001 Phys. Rev. D63 062005

[8] Pati M E and Will C M 2002 Phys. Rev. D65 104008

[9] Jaranowski P and Schafer G 1997 Phys. Rev. D55 4712

[10] Jaranowski P and Schäfer G 1998 Phys. Rev. D57 7274; 2001 Erratum-ibid. D63 029902

[11] Damour T, Jaranowski P and Schäfer G 2001 Phys. Lett. B513 147

[12] Blanchet L 2002 Living Rev. Relativ. 53

[13] Jaranowski P and Schäfer G 1999 Phys. Rev. D60 124003

[14] Damour T, Jaranowski P and Schäfer G 2001 Phys. Rev. D62 021501; 2000 Erratum-ibid. D63 029903

[15] Damour T, Jaranowski P and Schäfer G 2001 Phys. Rev. D63 044021; 2002 Erratum-ibid. D66 029901

[16] de Andrade V C, Blanchet L and Faye G 2001 Class. Quant. Grav. 18753

[17] Damour T 1983 in Gravitational Radiation, ed Deruelle N and Piran T (Amsterdam; NorthHolland) 59

[18] Itoh Y, Futamase T and Asada H 2000 Phys. Rev. D62 064002

[19] Itoh Y, Futamase T and Asada H 2001 Phys. Rev. D63 064038

[20] Futamase T 1987 Phys. Rev. D36 321

[21] Einstein A, Infeld L and Hoffmann B 1938 Ann. Math. 3965

[22] Itoh Y Preprint gr-qc/0310029

[23] Itoh Y and Futamase T 2003 Phys. Rev. D68 121501

[24] Blanchet L and Damour T 1988 Phys. Rev. D37 1410 